\title{
Gender-specific interactions of MTHFR C677T and MTRR A66G polymorphisms with overweight/obesity on serum lipid levels in a Chinese Han population
}

\author{
Xueyuan Zhi', Boyi Yang ${ }^{2}$, Shujun Fan', Yanxun Wang ${ }^{3}$, Jian Wei ${ }^{4}$, Quanmei Zheng ${ }^{1}$ and Guifan Sun ${ }^{1 *}$ (D)
}

\begin{abstract}
Background: Little is known regarding the interactions of methylenetetrahydrofolate reductase (MTHFR) C677T and methionine synthase reductase (MTRR) A66G polymorphisms with overweight/obesity on serum lipid profiles. The aim of the current study was to explore interactions between the two polymorphisms and overweight/obesity on four common lipid levels in a Chinese Han population and further to evaluate whether these interactions exhibit gender-specificity.
\end{abstract}

Methods: A total of 2239 participants (750 females and 1489 males) were enrolled into this study. The genotypes of the MTHFR C677T and MTRR A66G were determined by a TaqMan assay. Overweight and obesity were defined as a body mass index between 24 and 27.99 and $\geq 28 \mathrm{~kg} / \mathrm{m}^{2}$, respectively. The interactions were examined by factorial design covariance analysis, and further multiple comparisons were conducted by Bonferroni correction.

Results: There was no significant difference in the genotypic and allelic frequencies between females and males (MTHFR 677 T allele: $54.47 \%$ for females and $54.40 \%$ for males; MTRR 66G allele: $24.73 \%$ for females and $24.71 \%$ for males). Interaction between the MTHFR C677T polymorphism and overweight/obesity on serum triglyceride levels, and interaction between the MTRR A66G polymorphism and overweight/obesity on serum high-density lipoprotein cholesterol levels were detected in women $(P=0.015$ and $P=0.056$, respectively). For female subjects with overweight/obesity, the serum triglyceride levels in MTHFR 677T genotype [1.09 (0.78-1.50) mmol/L] were significantly higher as compared with MTHFR 677CC genotype [0.90 (0.60-1.15) $\mathrm{mmol} / \mathrm{L}, P=0.007$, and the MTRR 66GG genotype carriers had higher serum high-density lipoprotein cholesterol levels than those with MTRR 66AG genotype (1.46 \pm 0.50 vs. $1.19 \pm 0.31 \mathrm{mmol} / \mathrm{L}, P=0.058)$. Furthermore, in male subjects with overweight/obesity, the MTHFR 677CT genotype carriers had higher low-density lipoprotein cholesterol levels than those with MTHFR 677T genotype ( $2.96 \pm 1.07$ vs. $2.74 \pm 0.88 \mathrm{mmol} / \mathrm{L}, P=0.015$ ).

Conclusions: Our results indicate that there exist interactive effects of the MTHFR C677T and MTRR A66G polymorphisms with overweight/obesity on some lipid traits in Chinese Han population, and the effects were gender-specific.

Keywords: MTHFR C677T, MTRR A66G, Overweight, Obesity, Lipid, Interaction, Gender-specificity

\footnotetext{
* Correspondence: gfsun@cmu.edu.cn

${ }^{1}$ Research Center of Environment and Non-Communicable Disease, School of

Public Health, China Medical University, No.77 Puhe Road, Shenyang North

New Area, Shenyang 110122, People's Republic of China

Full list of author information is available at the end of the article
} 


\section{Background}

Dyslipidemia, serving as a crucial risk factor for cardiovascular diseases, has become a serious public health problem worldwide because of its high prevalence [1-3]. The etiology of dyslipidemia is complicated, and it is generally agreed that serum lipid concentrations are influenced by both genetic and environmental factors as well as their interactions [4-7].

Previous studies indicated that methylenetetrahydrofolate reductase (MTHFR) C677T and methionine synthase reductase (MTRR) A66G polymorphisms are significantly associated with serum lipid levels [8-11]. MTHFR catalyzes the reduction of methylenetetrahydrofolate to methyltetrahydrofolate, a key methyl donor for homocysteine remethylation to methionine [12]. At the $677 \mathrm{bp}$ of MTHFR gene, a $\mathrm{C}$ to $\mathrm{T}$ switch $(\mathrm{C} 677 \mathrm{~T})$ has been verified to reduce its enzyme activity, leading to accumulation of homocysteine [13-15]. MTRR is responsible for maintaining adequate levels of activated cobalamin, an indispensable cofactor for homocysteine remethylation. The MTRR A66G polymorphism results in difference in its enzyme expression and function, and further affects plasma homocysteine levels [16]. High homocysteine levels have been implicated to be associated with lipid change in numerous epidemiological studies [17-20]. Research with animal and cell models also demonstrated that high homocysteine concentrations interfere with some lipid parameters metabolism [21-24]. These links suggest that the MTHFR C677T and MTRR A66G polymorphisms may affect lipid concentrations by altering homocysteine levels.

However, not all prior studies have found significant correlations between the two polymorphisms and serum lipid levels. The inconsistency among studies may be partly due to different environmental factors that interact with gene to regulate serum lipid levels. Overweight/ obesity is a well-established modifiable contributor to the development of dyslipidemia [25-27]. Research on the interactions of the MTHFR C677T and MTRR A66G polymorphisms with overweight/obesity may provide valuable insight into the inconsistency among prior studies. However, information on the interactions of the two polymorphisms and overweight/obesity on serum lipid profiles is scarce. In a systematic Medline search, we found only one related study. In that study, Yin et al. observed significant interactions between the MTHFR C677T polymorphism and overweight/obesity on some lipid traits in Chinese Bai Ku Yao population [5]. Whether this interaction still exists in Chinese Han population remains elusive.

In addition, gender differences in lipid and lipoprotein metabolism have been well documented [28-31]. However, the underlying mechanism is unclear. Despite some advances in the identification of sex-specific genetic association with plasma lipid levels [11, 32, 33], few studies have explored the modifying effects of sex in the interactions between single nucleotide polymorphisms (SNPs) and overweight/obesity on lipid concentrations. Such exploration may provide valuable insight into the factors causing gender difference in the plasma lipid profile.

This study, therefore, was conducted to explore the interactions of the MTHFR C677T and MTRR A66G polymorphisms with overweight/obesity on four common lipid traits in a Chinese Han population and further to assess whether these interactions exhibit gender-specificity.

\section{Methods \\ Study population}

From October 2008 to February 2011, a total of 2239 individuals (750 females, $33.50 \%$ and 1489 males, $66.50 \%)$ were recruited into our study from an unselected population taking a regular health examination at the physical examination center of Dagang Oil Field General Hospital. Subjects with a history of gastrointestinal, thyroid, chronic liver, renal diseases, or diabetes mellitus were excluded. None of these subjects were treated with a lipid-lowering diet or drugs known to affect serum lipid levels within 4 weeks before the study. There was no blood relationship among all the selected subjects, which were of Han nationality living in Dagang district in Tianjin. Age of the participants ranged from 22 to 78 years with the mean age of $45.38 \pm 9.40$ years in females and $47.37 \pm 10.10$ years in males, respectively.

\section{Clinical measurements and laboratory tests}

National standard techniques were used to measure height and body weight, and body mass index (BMI) was calculated as weight in kilograms divided by the square of height in meters $\left(\mathrm{Kg} / \mathrm{m}^{2}\right)$. BMI $<24,24-27.99$ and $\geq 28$ was classified as normal weight, overweight and obesity, respectively [34]. Blood pressure was measured three times while study subjects were in the sitting position after 15 min of rest and the three measurements were averaged for analysis. After an overnight fast, venous blood samples and buccal cell samples were collected from all the participants for biochemical analysis and genetic analysis, respectively. The levels of triglyceride (TG), total cholesterol (TC), high-density lipoprotein cholesterol (HDL-C), lowdensity lipoprotein cholesterol (LDL-C) and fasting blood glucose (FBG) in blood samples were measured by enzymatic method on a Hitachi Autoanalyzer (Type 7170A; Hitachi Ltd., Tokyo, Japan) in Dagang Oil Field General Hospital.

\section{Genotyping}

Genomic DNA was extracted from buccal samples using the QIAamp DNA Mini Kit (Qiagen, Valencia, CA, USA). The genotypes of the MTHFR C677T and MTRR A66G were determined by a TaqMan assay, which has been described in our previous paper [35]. 


\section{Statistical analysis}

All statistical analyses were carried out using SAS version 9.2 (SAS Institute, Cary, NC, USA) and two-sided $P<0.05$ was considered to be statistically significant. Data were expressed as mean \pm standard deviation or median (interquartile range) for quantitative variables and as $n$ (\%) for qualitative variables. Variables with skewed distribution (serum TG) were log transformed before analysis. Genotypic and allelic frequencies were calculated via direct counting. The distributions of genotypes were analyzed for deviation from Hardy-Weinberg equilibrium using Chi-square test. Comparisons of difference between the female and male subjects with respect to general characteristics and genotype distribution were performed by two-sample $t$ test and Chi-square test, respectively (the difference in serum TG levels was determined by the Wilcoxon two-sample test). The interaction between overweight/obesity and two studied polymorphisms on serum lipid levels was tested by using a factorial design covariance analysis after controlling for age, and further multiple comparisons were conducted by Bonferroni correction.

\section{Results}

General characteristics and genotype distribution

Table 1 summarizes the basic characteristics and genotype distributions of the study population. Totally, 2239 participants were analyzed, of which 1489 (66.50\%) were male. Among the study population, females were significantly younger than males $(P<0.001)$. Compared with female subjects, the males had higher BMI and higher levels of systolic blood pressure, diastolic blood pressure, FBG, serum TG, TC and LDL-C (All $P<0.05$ ), whereas the serum HDL-C levels was lower in men than in women $(P<0.001)$.

The genotype distributions of both the MTHFR C677T and MTRR A66G polymorphisms were in accordance with Hardy-Weinberg equilibrium (both $P>0.05$ ) and were consistent with those previously reported for Tianjin population [35]. The frequency of the MTHFR 677CC, CT and TT genotypes was $22.00,47.07$ and $30.93 \%$ in females, and 21.49, 48.22 and $30.29 \%$ in males. As for the MTRR A66G polymorphism, the frequency of AA, AG and GG genotypes was $57.60,35.33$ and $7.07 \%$ in women, and 57.29, 36.00 and $6.72 \%$ in men. There was no significant difference between females and males in terms of the genotypic

Table 1 General characteristics and genotype distribution

\begin{tabular}{|c|c|c|c|c|}
\hline Variables & Total $(n=2239)$ & Female $(n=750)$ & Male $(n=1489)$ & $P$ \\
\hline Age, year & $46.70 \pm 9.92$ & $45.38 \pm 9.40$ & $47.37 \pm 10.10$ & $<0.001$ \\
\hline Height, cm & $168.37 \pm 7.67$ & $161.80 \pm 5.67$ & $171.68 \pm 6.28$ & $<0.001$ \\
\hline Weight, kg & $71.25 \pm 12.50$ & $61.60 \pm 9.50$ & $76.11 \pm 10.91$ & $<0.001$ \\
\hline BMI, $\mathrm{kg} / \mathrm{m}^{2}$ & $25.03 \pm 3.52$ & $23.52 \pm 3.36$ & $25.80 \pm 3.34$ & $<0.001$ \\
\hline $\mathrm{TG}^{\mathrm{a}}, \mathrm{mmol} / \mathrm{L}$ & $1.00(0.67-1.55)$ & $0.75(0.53-1.15)$ & $1.18(0.77-1.76)$ & $<0.001$ \\
\hline $\mathrm{TC}, \mathrm{mmol} / \mathrm{L}$ & $4.98 \pm 0.98$ & $4.91 \pm 0.98$ & $5.01 \pm 0.97$ & 0.021 \\
\hline $\mathrm{HDL}-\mathrm{C}, \mathrm{mmol} / \mathrm{L}$ & $1.22 \pm 0.33$ & $1.32 \pm 0.37$ & $1.16 \pm 0.29$ & $<0.001$ \\
\hline $\mathrm{LDL}-\mathrm{C}, \mathrm{mmol} / \mathrm{L}$ & $2.77 \pm 0.96$ & $2.71 \pm 0.94$ & $2.79 \pm 0.98$ & 0.050 \\
\hline $\mathrm{SBP}, \mathrm{mmHg}$ & $130.26 \pm 19.60$ & $123.73 \pm 18.52$ & $133.54 \pm 19.31$ & $<0.001$ \\
\hline $\mathrm{DBP}, \mathrm{mmHg}$ & $82.45 \pm 13.05$ & $77.28 \pm 11.01$ & $85.05 \pm 13.23$ & $<0.001$ \\
\hline $\mathrm{FBG}, \mathrm{mmol} / \mathrm{L}$ & $5.31 \pm 1.30$ & $5.07 \pm 0.95$ & $5.43 \pm 1.20$ & $<0.001$ \\
\hline \multicolumn{5}{|l|}{ MTHFR C677T } \\
\hline CC & $485(21.67)$ & $165(22.00)$ & $320(21.49)$ & - \\
\hline $\mathrm{CT}$ & $1071(47.83)$ & $353(47.07)$ & 718 (48.22) & - \\
\hline$\pi$ & $683(30.50)$ & 232 (30.93) & $451(30.29)$ & 0.875 \\
\hline C allele & $2041(45.58)$ & $683(45.53)$ & $1358(45.60)$ & - \\
\hline T allele & $2437(54.42)$ & 817 (54.47) & $1620(54.40)$ & 0.966 \\
\hline \multicolumn{5}{|l|}{ MTRR A66G } \\
\hline AA & $1285(57.39)$ & $432(57.60)$ & $853(57.29)$ & - \\
\hline$A G$ & 801 (35.77) & 265 (35.33) & $536(36.00)$ & - \\
\hline GG & 153 (6.83) & $53(7.07)$ & $100(6.72)$ & 0.923 \\
\hline A allele & 3371 (75.28) & $1129(75.27)$ & $2242(75.29)$ & - \\
\hline G allele & $1107(24.72)$ & 371 (24.73) & 736 (24.71) & 0.989 \\
\hline
\end{tabular}

$B M I$ body mass index, TG triglyceride, $T C$ total cholesterol, $H D L-C$ high-density lipoprotein cholesterol, $L D L-C$ low-density lipoprotein cholesterol, $S B P$ systolic blood pressure, DBP diastolic blood pressure, FBG fasting blood glucose, MTHFR methylenetetrahydrofolate reductase, MTRR methionine synthase reductase

${ }^{a}$ The value of TG was presented as median (interquartile range), and the difference in serum TG levels was determined by the Wilcoxon two-sample test 
frequencies of the MTHFR C677T and MTRR A66G polymorphisms (both $P>0.05$ ).

\section{Genotypes and serum lipid levels}

For total participants, no significant correlation was found between the MTHFR C677T polymorphism and serum lipid levels either in normal weight group or overweight/ obesity group (Table 2). When stratified by gender, the MTHFR 677TT genotype was associated with higher serum TG levels in female subjects with overweight/obesity $(P=0.007)$. For male subjects with overweight/obesity, the MTHFR $677 \mathrm{CT}$ genotype carriers had significantly higher LDL-C levels than the MTHFR 677TT genotype carriers $\operatorname{did}(P=0.018)$. However, in either women or men, no significant difference was detected in the four lipid parameters for normal weight subjects bearing different genotypes of the MTHFR C677T polymorphism.
Subjects carrying the MTRR 66GG genotype had higher serum HDL-C levels than those with MTRR 66AA or AG genotypes in overweight/obese individuals (Table $3, P=0.035$ and $P=0.084$, respectively). When stratified by gender, similar association only exists in female subjects with overweight/obesity.

\section{Overweight/obesity and serum lipid levels}

As listed in Table 2, overweight/obesity group had higher TG and lower HDL-C levels as compared with normal weight group in MTHFR 677CC, CT and TT genotype carriers (All $P<0.05$ ), whereas significant difference of TC and LDL-C levels between the two groups were only found in subjects with MTHFR 677CT genotype (Both $P<0.05$ ). Similarly, overweight/obesity group had higher TG levels than normal weight group in MTRR 66AA, AG and GG genotype carriers (Table 3,

Table 2 Interaction of MTHFR C677T polymorphism with overweight/obesity on serum lipid levels

\begin{tabular}{|c|c|c|c|c|c|c|c|}
\hline BMI & Genotype & $n$ & $\mathrm{TG}(\mathrm{mmol} / \mathrm{L})$ & $\operatorname{Lg}(\mathrm{TG})$ & $\mathrm{TC}(\mathrm{mmol} / \mathrm{L})$ & $\mathrm{HDL}-\mathrm{C}(\mathrm{mmol} / \mathrm{L})$ & $\mathrm{LDL}-\mathrm{C}(\mathrm{mmol} / \mathrm{L})$ \\
\hline \multicolumn{8}{|l|}{ Total } \\
\hline \multirow[t]{3}{*}{$<24$} & CC & 198 & $0.71(0.53-1.07)$ & $-0.10 \pm 0.25$ & $4.83 \pm 1.07$ & $1.32 \pm 0.37$ & $2.65 \pm 0.97$ \\
\hline & $C T$ & 438 & $0.76(0.59-1.17)$ & $-0.08 \pm 0.24$ & $4.86 \pm 1.01$ & $1.31 \pm 0.34$ & $2.68 \pm 0.95$ \\
\hline & $\Pi$ & 249 & $0.69(0.49-1.02)$ & $-0.12 \pm 0.27$ & $4.80 \pm 0.95$ & $1.34 \pm 0.36$ & $2.63 \pm 0.92$ \\
\hline \multirow[t]{3}{*}{$\geq 24$} & CC & 287 & $1.15(0.78-1.81)$ & $0.08 \pm 0.26^{a, b, c}$ & $5.02 \pm 0.89$ & $1.17 \pm 0.32^{a, b, c}$ & $2.79 \pm 0.89$ \\
\hline & $C T$ & 633 & $1.25(0.86-1.85)$ & $0.11 \pm 0.27^{a, b, c}$ & $5.12 \pm 0.98^{a, b, c}$ & $1.14 \pm 0.28^{a, b}, c$ & $2.92 \pm 1.04^{a, b, c}$ \\
\hline & $\pi$ & 434 & $1.25(0.86-1.77)$ & $0.11 \pm 0.27^{a, b}, c$ & $5.02 \pm 0.93$ & $1.14 \pm 0.28^{a, b, c}$ & $2.74 \pm 0.91$ \\
\hline$P^{*}$ interaction & & & - & 0.268 & 0.668 & 0.268 & 0.296 \\
\hline \multicolumn{8}{|l|}{ Female } \\
\hline \multirow[t]{3}{*}{$<24$} & CC & 104 & $0.65(0.50-0.89)$ & $-0.16 \pm 0.23$ & $4.82 \pm 1.03$ & $1.39 \pm 0.38$ & $2.66 \pm 0.93$ \\
\hline & $C T$ & 224 & $0.71(0.53-0.94)$ & $-0.13 \pm 0.24$ & $4.85 \pm 0.89$ & $1.36 \pm 0.36$ & $2.68 \pm 0.93$ \\
\hline & $\pi$ & 142 & $0.63(0.44-0.90)$ & $-0.16 \pm 0.28$ & $4.82 \pm 1.01$ & $1.40 \pm 0.39$ & $2.65 \pm 0.98$ \\
\hline \multirow[t]{3}{*}{$\geq 24$} & CC & 61 & $0.90(0.60-1.15)$ & $-0.07 \pm 0.22$ & $5.13 \pm 0.98$ & $1.28 \pm 0.36$ & $2.90 \pm 0.87$ \\
\hline & $\mathrm{CT}$ & 129 & $0.99(0.65-1.43)$ & $0.00 \pm 0.23^{a, b, c}$ & $5.04 \pm 0.98$ & $1.23 \pm 0.31^{a, b, c}$ & $2.78 \pm 0.90$ \\
\hline & $\pi$ & 90 & $1.09(0.78-1.50)$ & $0.05 \pm 0.27^{a, b}, c, d$ & $4.94 \pm 1.10$ & $1.17 \pm 0.32^{a, b, c}$ & $2.71 \pm 1.01$ \\
\hline$P^{*}{ }_{\text {interaction }}$ & & & - & 0.015 & 0.812 & 0.161 & 0.750 \\
\hline \multicolumn{8}{|l|}{ Male } \\
\hline \multirow[t]{3}{*}{$<24$} & $\mathrm{CC}$ & 94 & $0.81(0.57-1.25)$ & $-0.05 \pm 0.25$ & $4.83 \pm 1.12$ & $1.24 \pm 0.35$ & $2.65 \pm 1.01$ \\
\hline & $C T$ & 214 & $0.88(0.65-1.32)$ & $-0.03 \pm 0.24$ & $4.87 \pm 1.12$ & $1.26 \pm 0.30$ & $2.68 \pm 0.97$ \\
\hline & $\pi$ & 107 & $0.79(0.56-1.18)$ & $-0.07 \pm 0.25$ & $4.77 \pm 0.88$ & $1.26 \pm 0.31$ & $2.60 \pm 0.83$ \\
\hline \multirow[t]{3}{*}{$\geq 24$} & CC & 226 & $1.24(0.87-1.95)$ & $0.12 \pm 0.26^{a, b, c}$ & $4.99 \pm 0.87$ & $1.14 \pm 0.30^{a, b}, c$ & $2.76 \pm 0.89$ \\
\hline & $C T$ & 504 & $1.32(0.91-2.01)$ & $0.14 \pm 0.27^{a, b, c}$ & $5.14 \pm 0.98^{b, c}$ & $1.12 \pm 0.27^{a, b, c}$ & $2.96 \pm 1.07^{a, b, c}$ \\
\hline & $\pi$ & 344 & $1.28(0.86-1.79)$ & $0.12 \pm 0.26^{a, b, c}$ & $5.04 \pm 0.89$ & $1.13 \pm 0.26^{a, b, c}$ & $2.74 \pm 0.88^{e}$ \\
\hline$P^{*}$ interaction & & & - & 0.625 & 0.747 & 0.747 & 0.399 \\
\hline
\end{tabular}

$B M I$ body mass index, MTHFR methylenetetrahydrofolate reductase *Adjusted for age

${ }^{\mathrm{a}} P<0.05$ in comparison with $\mathrm{CC}$ genotype of the $\mathrm{BMI}<24$ subgroup ${ }^{\mathrm{b}} P<0.05$ in comparison with $C \mathrm{CT}$ genotype of the $\mathrm{BMI}<24$ subgroup ${ }^{c} P<0.05$ in comparison with $\Pi$ genotype of the $B M I<24$ subgroup ${ }^{\mathrm{d}} P<0.05$ in comparison with $C C$ genotype of the $\mathrm{BMI} \geq 24$ subgroup ${ }^{\mathrm{e}} P<0.05$ in comparison with CT genotype of the BMI $\geq 24$ subgroup 
Table 3 Interaction of MTRR A66G polymorphism with overweight/obesity on serum lipid levels

\begin{tabular}{|c|c|c|c|c|c|c|c|}
\hline $\mathrm{BMI}$ & Genotype & $n$ & $\mathrm{TG}(\mathrm{mmol} / \mathrm{L})$ & $\operatorname{Lg}(\mathrm{TG})$ & $\mathrm{TC}(\mathrm{mmol} / \mathrm{L})$ & $\mathrm{HDL}-\mathrm{C}(\mathrm{mmol} / \mathrm{L})$ & $\mathrm{LDL}-\mathrm{C}(\mathrm{mmol} / \mathrm{L})$ \\
\hline \multicolumn{8}{|l|}{ Total } \\
\hline \multirow[t]{3}{*}{$<24$} & $\mathrm{AA}$ & 511 & $0.73(0.54-1.10)$ & $-0.10 \pm 0.24$ & $4.84 \pm 1.01$ & $1.31 \pm 0.36$ & $2.68 \pm 0.96$ \\
\hline & $A G$ & 309 & $0.72(0.53-1.07)$ & $-0.10 \pm 0.27$ & $4.84 \pm 1.02$ & $1.35 \pm 0.34$ & $2.64 \pm 0.93$ \\
\hline & GG & 65 & $0.77(0.59-1.20)$ & $-0.07 \pm 0.25$ & $4.82 \pm 0.90$ & $1.31 \pm 0.36$ & $2.62 \pm 0.90$ \\
\hline \multirow[t]{3}{*}{$\geq 24$} & $\mathrm{AA}$ & 774 & $1.24(0.86-1.87)$ & $0.11 \pm 0.28^{a, b}, c$ & $5.07 \pm 0.93^{a, b}$ & $1.14 \pm 0.28^{a, b, c}$ & $2.84 \pm 0.96^{b}$ \\
\hline & $A G$ & 492 & $1.21(0.84-1.69)$ & $0.09 \pm 0.25^{a, b, c}$ & $5.03 \pm 0.98$ & $1.14 \pm 0.28^{\mathrm{a}, \mathrm{b}, \mathrm{c}}$ & $2.81 \pm 0.99$ \\
\hline & GG & 88 & $1.21(0.77-2.14)$ & $0.11 \pm 0.27^{a, b, c}$ & $5.22 \pm 0.95$ & $1.25 \pm 0.33^{\mathrm{d}, \mathrm{e}}$ & $2.95 \pm 1.02$ \\
\hline$P^{*}$ interaction & & & - & 0.438 & 0.688 & 0.050 & 0.608 \\
\hline \multicolumn{8}{|l|}{ Female } \\
\hline \multirow[t]{3}{*}{$<24$} & $\mathrm{AA}$ & 277 & $0.69(0.49-0.94)$ & $-0.15 \pm 0.24$ & $4.80 \pm 0.97$ & $1.37 \pm 0.38$ & $2.65 \pm 0.97$ \\
\hline & $A G$ & 158 & $0.66(0.48-0.90)$ & $-0.15 \pm 0.27$ & $4.88 \pm 0.93$ & $1.39 \pm 0.36$ & $2.70 \pm 0.91$ \\
\hline & GG & 35 & $0.68(0.52-0.89)$ & $-0.14 \pm 0.22$ & $4.88 \pm 1.00$ & $1.39 \pm 0.41$ & $2.64 \pm 0.90$ \\
\hline \multirow[t]{3}{*}{$\geq 24$} & $\mathrm{AA}$ & 155 & $0.96(0.65-1.41)$ & $-0.00 \pm 0.25^{a, b}$ & $5.01 \pm 0.97$ & $1.22 \pm 0.31^{a, b}$ & $2.72 \pm 0.90$ \\
\hline & $A G$ & 107 & $1.00(0.70-1.46)$ & $0.01 \pm 0.25^{a, b}$ & $5.01 \pm 1.07$ & $1.19 \pm 0.31^{a, b, c}$ & $2.84 \pm 0.96$ \\
\hline & GG & 18 & $0.96(0.57-1.19)$ & $-0.02 \pm 0.27$ & $5.31 \pm 1.12$ & $1.46 \pm 0.50^{f}$ & $3.03 \pm 1.01$ \\
\hline$P^{*}$ interaction & & & - & 0.598 & 0.937 & 0.056 & 0.593 \\
\hline \multicolumn{8}{|l|}{ Male } \\
\hline \multirow[t]{3}{*}{$<24$} & $\mathrm{AA}$ & 234 & $0.83(0.63-1.24)$ & $-0.05 \pm 0.23$ & $4.88 \pm 1.06$ & $1.23 \pm 0.32$ & $2.71 \pm 0.95$ \\
\hline & $A G$ & 151 & $0.82(0.57-1.25)$ & $-0.06 \pm 0.25$ & $4.79 \pm 1.11$ & $1.29 \pm 0.31$ & $2.57 \pm 0.95$ \\
\hline & GG & 30 & $1.02(0.63-1.47)$ & $0.02 \pm 0.26$ & $4.75 \pm 0.80$ & $1.22 \pm 0.28$ & $2.60 \pm 0.92$ \\
\hline \multirow[t]{3}{*}{$\geq 24$} & AA & 619 & $1.30(0.90-1.97)$ & $0.14 \pm 0.27^{a, b}$ & $5.09 \pm 0.92^{b}$ & $1.12 \pm 0.27^{a, b}$ & $2.87 \pm 0.97^{b}$ \\
\hline & $A G$ & 385 & $1.27(0.87-1.78)$ & $0.11 \pm 0.25^{a, b}$ & $5.04 \pm 0.95$ & $1.13 \pm 0.28^{a, b}$ & $2.81 \pm 1.00$ \\
\hline & GG & 70 & $1.32(0.82-2.20)$ & $0.14 \pm 0.26^{a, b}$ & $5.20 \pm 0.91$ & $1.19 \pm 0.25$ & $2.93 \pm 1.03$ \\
\hline$P^{*}$ interaction & & & - & 0.430 & 0.606 & 0.109 & 0.649 \\
\hline
\end{tabular}

$B M I$ body mass index, MTRR methionine synthase reductase ${ }^{*}$ Adjusted for age

${ }^{\text {a }} P<0.05$ in comparison with $\mathrm{AA}$ genotype of the $\mathrm{BMI}<24$ subgroup

${ }^{\mathrm{b}} P<0.05$ in comparison with $\mathrm{AG}$ genotype of the $\mathrm{BMI}<24$ subgroup

${ }^{c} P<0.05$ in comparison with GG genotype of the $B M l<24$ subgroup

${ }^{\mathrm{d}} P<0.05$ in comparison with AA genotype of the $\mathrm{BMI} \geq 24$ subgroup

${ }^{\mathrm{e}} P=0.084$ in comparison with $A G$ genotype of the $B M I \geq 24$ subgroup

${ }^{f} P=0.058$ in comparison with AG genotype of the BMI $\geq 24$ subgroup

All $P<0.05)$. The correlation between overweight/obesity and higher TC levels was only found in subjects with MTRR 66AA genotype $(P<0.05)$. HDL-C levels were lower in MTRR 66AA and AG genotypes carriers with overweight/obesity than in those with normal weight (Both $P<0.05$ ). Stratified analyses by gender showed similar results (Tables 2 and 3).

\section{Interactions of polymorphism with overweight/obesity on serum lipid levels}

Participants were divided into six groups according to their genotypes and BMI. Then we analyzed the interaction and compared the difference of serum lipid levels among six groups.

In total population, no significant interaction between the MTHFR C677T polymorphism and overweight/obesity on lipid traits was observed (Table 2).
However, this polymorphism interacts with overweight/ obesity to influence serum TG levels among female subjects $(P=0.015)$. The MTHFR 677TT genotype carriers with overweight/obesity had higher serum TG levels than other groups; the differences were statistically significant except for that between the MTHFR 677TT genotype carriers with overweight/obesity and the MTHFR 677CT genotype carriers with overweight/obesity.

For the MTRR A66G polymorphism, we detected a borderline significant interaction between this polymorphism and overweight/obesity on serum HDL-C levels $(P=0.050$, Table 3$)$. When stratified by gender, this borderline significant interaction only existed in females $(P=0.056)$. The MTRR 66AG genotype carriers with overweight/obesity had lower serum HDL-C levels than the others; the differences were statistically significant except for that between the MTRR 66AG genotype 
carriers with overweight/obesity and the MTRR 66AA genotype carriers with overweight/obesity.

\section{Discussion}

In the current study, interaction between the MTHFR C677T polymorphism and overweight/obesity on serum TG levels, and interaction between the MTRR A66G polymorphism and overweight/obesity on serum HDL-C levels were detected in women but not in men. We found that the MTHFR 677TT genotype had detrimental effects on serum TG levels and the MTRR 66GG genotype had favourable effects on serum HDL-C levels in female subjects with overweight/obesity. Furthermore, in male subjects with overweight/obesity, the MTHFR 677CT genotype carriers had higher LDL-C levels than TT genotype.

Many previous studies have explored the relationship between the MTHFR C677T polymorphism and serum or plasma lipid profiles in humans, with some indicating that the $\mathrm{T}$ allele was associated with unfavourable lipid profiles [8-11, 17, 36-39], whereas the others reported null association [40-43]. An interesting study by Zhang et al. indicated that the MTHFR $677 \mathrm{~T}$ allele significantly increases the serum levels of TC and LDL-C in both Chinese Bai Ku Yao and Han populations [10]. Among Polish elderly women, the MTHFR 677CT/TT genotypes were associated with higher TC and lower HDL-C levels as compared with CC genotype [36]. According to Huang et al. [39], hyperlipidemia patients with MTHFR 677CT/TT genotypes had higher TG concentrations than those with $\mathrm{CC}$ genotype. However, $\mathrm{Li}$ and coworkers failed to find any significant correlations between this polymorphism and four common lipid traits (TG, TC, HDL-C and LDL-C) in hypertensive patients [42]. Various factors including gender may modulate the association between this polymorphism and lipid levels and then lead to inconsistency among these studies.

For instance, Chen et al. initially failed to find significant difference in the four lipid measures (TG, TC, HDL-C and LDL-C) among the MTHFR C677T genotypes in longevous individuals [37]. However, when gender was taken into consideration, the MTHFR $677 \mathrm{~T}$ allele carriers of females but not males were found to have significantly higher levels of TC and LDL-C than $\mathrm{CC}$ genotype. Similar finding was also reported in essential hypertensive patients [11]. That study found that female but not male hypertensive patients with MTHFR 677TT genotype had higher LDL-C levels than those with CC genotype. In contrast, Zhang et al. found that significant association between serum LDL-C levels and this polymorphism only exists in males in both Chinese Bai Ku Yao and Han populations [10]. In agreement with the findings of Zhang et al., our results indicated that significant correlation between this variant and serum LDL-C levels only existed in males with overweight/ obesity. In addition, we also found that MTHFR 677TT genotype was associated with higher serum TG levels in females with overweight/obesity. Though heterogeneity by sex for lipid levels had been previously reported for other SNPs [33], the underlying mechanisms for the gender-specific association are unclear. It may be partly resulted from hormone action in combination with social and behavioral differences between men and women [29].

Regarding the MTRR A66G polymorphism, our results indicated that MTRR 66GG genotype was associated with higher HDL-C levels in female subjects with overweight/obesity. Jiang et al. reported that the serum TC and LDL-C levels were lower in MTRR 66GG genotype than in MTRR 66AA genotype among Chinese hypertensive patients [11]. Similarly, when stratified by gender, these correlations were only evident in women. But in another sample of Chinese hypertensive patients, a marginally significant association was found between MTRR 66GG genotype and elevated serum TC levels [42]. Our group previously conducted a case-control study to investigate the association of this genetic variant with metabolic syndrome [44], and found that MTRR 66GG genotype was associated with increased risk of high TG (TG $\geq 1.7 \mathrm{mmol} / \mathrm{L}$ ), while no significant relationship was detected between this polymorphism and low HDL-C (HDL-C $<1.03 \mathrm{mmol} / \mathrm{L}$ for males and $<1.30 \mathrm{mmol} / \mathrm{L}$ for females). However, Misiak et al. found there was no significant relationship between this polymorphism and TG or HDL-C levels in both first-episode schizophrenia patients and healthy controls [45]. The gender-specific effect of the MTRR A66G polymorphism on blood lipid profiles was not well explored. Moreover, unlike our study selecting general population without any severe diseases, the studies mentioned above were conducted in specific populations with various disorders such as hypertension [11, 42], metabolic syndrome [44] and schizophrenia [45], which may be a crucial contributor for the difference between our results and the prior findings.

In addition, nutrition and dietary habits are also important factors which may modulate the relationship between the two polymorphisms and lipid profiles. It has been reported that many nutraceuticals are able to actively influence lipid profiles and effectively improve dyslipidemia. Furthermore, because of the ability to attack several biochemical pathways of lipid metabolism, nutraceuticals appear to have the potential to overcome the genetic variability of individuals [46]. Thus, future studies should take nutraceuticals into consideration when evaluating the influence of genetics on lipid levels.

Literature about interactions between the two studied polymorphisms and overweight/obesity on blood lipid profiles was limited. In a systematic Medline search, we only found one study that examined interaction between the MTHFR C677T polymorphism and overweight/ 
obesity on serum lipid levels [5]. In that study, Yin et al. observed that the MTHFR C677T polymorphism interacted with high BMI to influence serum levels of TG, TC and LDL-C in Chinese Bai Ku Yao population. We failed to find significant interaction between the MTHFR C677T polymorphism and overweight/obesity, whereas a borderline significant interaction between the MTRR A66G polymorphism and overweight/obesity on serum HDL-C levels was observed.

Considering the gender differences in lipid and lipoprotein metabolism, we further assessed whether the interactions exhibit gender-specificity. Then we found that the MTHFR 677TT genotype interacts with overweight/obesity to increase serum TG levels in females, a finding consistent with the result from Chinese Bai $\mathrm{Ku}$ Yao population [5]. Likewise, the borderline significant interaction between the MTRR A66G polymorphism and overweight/obesity was only evident in women. It is difficult to compare our findings with other studies, because, to the best of our knowledge, this is the first study reporting gender-specific interactions of the two polymorphisms with overweight/obesity on serum lipid levels. Although many studies have reported that certain lipid-related genetic variants interact with environmental factors, such as high BMI, alcohol consumption and cigarette smoking, to modify blood lipid profiles [7, 47-50], all of them were performed on women and men combined. If our gender-specific findings reflect true causal associations, it would indicate that women with the MTHFR 677TT or the MTRR 66AG genotypes are more susceptible to the negative influence from overweight/obesity on some lipid traits. Future work is needed to clarify the underlying mechanism and confirm or refute our findings.

In this study, we selected ethnically homogeneous population (Chinese Han), which would minimize population stratification bias. Despite the assets of the current study, several limitations should be considered. First of all, although the sample size was relatively large, the number of female subjects in some subgroup analyses was a bit small. Next, the limitation of generalization of our results was that we enrolled the study subjects from one hospital. However, genotype distributions of the two polymorphisms were in accordance with Hardy-Weinberg equilibrium and were also consistent with those previously reported for Tianjin population [35]. The evidence suggests that our study subjects still have certain representativeness. Finally, it is well known that serum lipid levels are influenced by both genetic and environmental factors as well as their interactions. We only investigated two polymorphisms and did not have data on other environmental factors, such as dietary habits, alcohol consumption and cigarette smoking. Thus, the role of other genes, other environmental exposures and other gene-environment interactions on serum lipid profiles remain to be determined.

\section{Conclusions}

In conclusion, we present the first study to date on the gender difference in the interactions of the MTHFR C677T and MTRR A66G polymorphisms with overweight/obesity on some serum lipid traits. Interaction between the MTHFR C677T polymorphism and overweight/ obesity on TG levels was only evident in women, as was interaction between the MTRR A66G polymorphism and overweight/obesity on HDL-C levels. In the future, well-designed studies are required to confirm or refute our findings.

\section{Abbreviations}

BMI: Body mass index; DBP: Diastolic blood pressure; FBG: Fasting blood glucose; HDL-C: High-density lipoprotein cholesterol; LDL-C: Low-density lipoprotein cholesterol; MTHFR: Methylenetetrahydrofolate reductase; MTRR: Methionine synthase reductase; SBP: Systolic blood pressure; TC: Total cholesterol; TG: Triglyceride

\section{Acknowledgements}

We gratefully acknowledge the assistance and cooperation of the faculty and staff of Dagang Oil Field General Hospital and thank all of the participants in our study.

\section{Funding}

Not applicable.

\section{Availability of data and materials}

The datasets generated during the present study are not publicly available, because detailed genetic information of each participant were included in these materials.

\section{Authors' contributions}

XZ and GS conceived the study design and wrote the paper. BY, YW and JW performed the experiments, collected the data, and performed the statistical analysis. SF and QZ were responsible for the quality control of data.

All authors read and approved the final manuscript.

\section{Competing interests}

The authors declare that they have no competing interests.

\section{Consent for publication}

Not applicable.

\section{Ethics approval and consent to participate}

The Ethics Review Committee of China Medical University approved this study (Shenyang, China; Identification Code: CMU62073024; 15 July 2008) and all procedures were conducted in accordance with the Declaration of Helsinki. All subjects were fully informed of the study aims and design and a written informed consent was obtained from each participant.

\section{Author details}

${ }^{1}$ Research Center of Environment and Non-Communicable Disease, School of Public Health, China Medical University, No.77 Puhe Road, Shenyang North New Area, Shenyang 110122, People's Republic of China. ${ }^{2}$ Guangzhou Key Laboratory of Environmental Pollution and Health Risk Assessment,

Department of Preventive Medicine, School of Public Health, Sun Yat-Sen University, Guangzhou 510080, China. ${ }^{3}$ Division of Molecular Preventive Medicine, Shanghai Institute of Targeted Therapy and Molecular Medicine, Shanghai 200433, China. ${ }^{4}$ Brain Disease Center, Tianjin Dagang Oil Field General Hospital, Tianjin 300280, China.

Received: 17 June 2016 Accepted: 16 October 2016 Published online: 28 October 2016 


\section{References}

1. Parish S, Offer A, Clarke R, Hopewell JC, Hill MR, Otvos JD, Armitage J, Collins R. Lipids and lipoproteins and risk of different vascular events in the MRC/BHF Heart Protection Study. Circulation. 2012;125:2469-78.

2. Wu J, Cheng X, Qiu L, Xu T, Zhu G, Han J, Xia L, Qin X, Cheng Q, Liu Q. Prevalence and Clustering of Major Cardiovascular Risk Factors in China: A Recent Cross-Sectional Survey. Medicine (Baltimore). 2016;95:e2712.

3. Sun GZ, Li Z, Guo L, Zhou Y, Yang HM, Sun YX. High prevalence of dyslipidemia and associated risk factors among rural Chinese adults. Lipids Health Dis. 2014;13:189.

4. Yin RX, Li YY, Liu WY, Zhang L, Wu JZ. Interactions of the apolipoprotein A5 gene polymorphisms and alcohol consumption on serum lipid levels. PLoS One. 2011;6:e17954

5. Yin RX, Wu DF, Miao L, Aung LH, Cao XL, Yan TT, Long XJ, Liu WY, Zhang L, Li M. Several genetic polymorphisms interact with overweight/obesity to influence serum lipid levels. Cardiovasc Diabetol. 2012;11:123.

6. Pitsavos C, Panagiotakos D, Trichopoulou A, Chrysohoou C, Dedoussis G, Chloptsios Y, Choumerianou D, Stefanadis C. Interaction between Mediterranean diet and methylenetetrahydrofolate reductase C677T mutation on oxidized low density lipoprotein concentrations: the ATTICA study. Nutr Metab Cardiovasc Dis. 2006;16:91-9.

7. Cole CB, Nikpay M, McPherson R. Gene-environment interaction in dyslipidemia. Curr Opin Lipidol. 2015;26:133-8.

8. Liu Y, Li K, Venners SA, Hsu YH, Jiang S, Weinstock J, et al. Individual and joint associations of methylenetetrahydrofolate reductase C677T genotype and plasma homocysteine with dyslipidemia in a Chinese population with hypertension. Clin Appl Thromb Hemost. 2015. doi: 10.1177/1076029615609686.

9. Petta S, Bellia C, Mazzola A, Cabibi D, Camma C, Caruso A, Di Marco V, Craxi A, Ciaccio M. Methylenetetrahydrofolate reductase homozygosis and low-density lipoproteins in patients with genotype 1 chronic hepatitis $C$. J Viral Hepat. 2012;19:465-72

10. Zhang L, Yin RX, Liu WY, Miao L, Wu DF, Aung LH, Hu XJ, Cao XL, Wu JZ, Pan SL. Association of methylenetetrahydrofolate reductase C677T polymorphism and serum lipid levels in the Guangxi Bai Ku Yao and Han populations. Lipids Health Dis. 2010;9:123.

11. Jiang S, Zhao R, Pan M, Venners SA, Zhong G, Hsu YH. Associations of MTHFR and MTRR polymorphisms with serum lipid levels in Chinese hypertensive patients. Clin Appl Thromb Hemost. 2014:20:400-10.

12. Goyette P, Sumner JS, Milos R, Duncan AM, Rosenblatt DS, Matthews RG, Rozen R. Human methylenetetrahydrofolate reductase: isolation of cDNA, mapping and mutation identification. Nat Genet. 1994;7:195-200.

13. Rozen R. Genetic predisposition to hyperhomocysteinemia: deficiency of methylenetetrahydrofolate reductase (MTHFR). Thromb Haemost. 1997;78:523-6.

14. Rozen R. Genetic modulation of homocysteinemia. Semin Thromb Hemost. 2000;26:255-61.

15. Frosst $P$, Blom HJ, Milos R, Goyette P, Sheppard CA, Matthews RG, Boers GJ, den Heijer M, Kluijtmans LA, van den Heuvel LP, Et A. A candidate genetic risk factor for vascular disease: a common mutation in methylenetetrahydrofolate reductase. Nat Genet. 1995;10:111-3.

16. Gaughan DJ, Kluijtmans LA, Barbaux S, McMaster D, Young IS, Yarnell JW, Evans A, Whitehead AS. The methionine synthase reductase (MTRR) A66G polymorphism is a novel genetic determinant of plasma homocysteine concentrations. Atherosclerosis. 2001;157:451-6.

17. Real JT, Martinez-Hervas S, Garcia-Garcia AB, Chaves FJ, Civera M, Ascaso JF, Carmena R. Association of C677T polymorphism in MTHFR gene, high homocysteine and low HDL cholesterol plasma values in heterozygous familial hypercholesterolemia. J Atheroscler Thromb. 2009;16:815-20.

18. Qujeq D, Omran TS, Hosini L. Correlation between total homocysteine, low-density lipoprotein cholesterol and high-density lipoprotein cholesterol in the serum of patients with myocardial infarction. Clin Biochem. 2001:34:97-101.

19. Mahalle N, Garg MK, Naik SS, Kulkarni MV. Study of pattern of dyslipidemia and its correlation with cardiovascular risk factors in patients with proven coronary artery disease. Ind J Endocrinol Metab. 2014;18:48-55.

20. Xiao Y, Zhang Y, LV X, Su D, Li D, Xia M, Qiu J, Ling W, Ma J. Relationship between lipid profiles and plasma total homocysteine, cysteine and the risk of coronary artery disease in coronary angiographic subjects. Lipids Health Dis. 2011;10:137

21. Woo CW, Siow YL, Pierce GN, Choy PC, Minuk GY, Mymin D, Karmin O. Hyperhomocysteinemia induces hepatic cholesterol biosynthesis and lipid accumulation via activation of transcription factors. Am J Physiol Endocrinol Metab. 2005:288:E1002-10.

22. Liao D, Tan H, Hui R, Li Z, Jiang X, Gaubatz J, Yang F, Durante W, Chan L, Schafer Al, Pownall HJ, Yang X, Wang H. Hyperhomocysteinemia decreases circulating high-density lipoprotein by inhibiting apolipoprotein A-I Protein synthesis and enhancing HDL cholesterol clearance. Circ Res. 2006:99:598-606.

23. Werstuck GH, Lentz SR, Dayal S, Hossain GS, Sood SK, Shi YY, Zhou J, Maeda N, Krisans SK, Malinow MR, Austin RC. Homocysteine-induced endoplasmic reticulum stress causes dysregulation of the cholesterol and triglyceride biosynthetic pathways. J Clin Invest. 2001;107:1263-73.

24. Karmin O, Lynn EG, Chung YH, Siow YL, Man RY, Choy PC. Homocysteine stimulates the production and secretion of cholesterol in hepatic cells. Biochim Biophys Acta. 1998;1393:317-24.

25. Kawada T. Body mass index is a good predictor of hypertension and hyperlipidemia in a rural Japanese population. Int J Obes Relat Metab Disord. 2002;26:725-9.

26. Klop B, Elte JW, Cabezas MC. Dyslipidemia in obesity: mechanisms and potential targets. Nutrients. 2013;5:1218-40.

27. Denke MA, Sempos CT, Grundy SM. Excess body weight. An underrecognized contributor to dyslipidemia in white American women. Arch Intern Med. 1994;154:401-10.

28. Johnson JL, Slentz CA, Duscha BD, Samsa GP, McCartney JS, Houmard JA, Kraus WE. Gender and racial differences in lipoprotein subclass distributions: the STRRIDE study. Atherosclerosis. 2004;176:371-7.

29. Wang X, Magkos F, Mittendorfer B. Sex differences in lipid and lipoprotein metabolism: it's not just about sex hormones. J Clin Endocrinol Metab. 2011;96:885-93.

30. Freedman DS, Otvos JD, Jeyarajah EJ, Shalaurova I, Cupples LA, Parise H, D'Agostino RB, Wilson PW, Schaefer EJ. Sex and age differences in lipoprotein subclasses measured by nuclear magnetic resonance spectroscopy: the Framingham Study. Clin Chem. 2004;50:1189-200.

31. Mittendorfer B. Sexual dimorphism in human lipid metabolism. J Nutr. 2005;135:681-6.

32. Aung LH, Yin RX, Wu DF, Wang W, Wu JZ, Liu CW. Sex-specific association of the zinc finger protein 259 rs2075290 polymorphism and serum lipid levels. Int J Med Sci. 2014:11:471-8.

33. Taylor KC, Carty CL, Dumitrescu L, Buzkova P, Cole SA, Hindorff L, Schumacher FR, Wilkens LR, Shohet RV, Quibrera PM, Johnson KC, Henderson BE, Haessler J, Franceschini N, Eaton CB, Duggan DJ, Cochran B, Cheng I, Carlson CS, Brown-Gentry K, Anderson G, Ambite JL, Haiman C, Le Marchand L, Kooperberg C, Crawford DC, Buyske S, North KE, Fornage M. Investigation of gene-by-sex interactions for lipid traits in diverse populations from the population architecture using genomics and epidemiology study. BMC Genet. 2013;14:33.

34. Zhou BF. Predictive values of body mass index and waist circumference for risk factors of certain related diseases in Chinese adults: study on optimal cut-off points of body mass index and waist circumference in Chinese adults. Asia Pac J Clin Nutr. 2002;11:S685-93.

35. Yang B, Liu Y, Li Y, Fan S, Zhi X, Lu X, Wang D, Zheng Q, Wang Y, Wang Y, Sun G. Geographical distribution of MTHFR C677T, A1298C and MTRR A66G gene polymorphisms in China: findings from 15357 adults of Han nationality. PLoS One. 2013;8:e57917.

36. Chmurzynska A, Malinowska AM, Twardowska-Rajewska J, Gawecki J. Elderly women: homocysteine reduction by short-term folic acid supplementation resulting in increased glucose concentrations and affecting lipid metabolism (C677T MTHFR polymorphism). Nutrition. 2013;29:841-4

37. Chen NY, Liu CW, Du LL, Xiao LP, Ge L, Wang YY, Wei Z, Wu HY, Luo CY, Liang L, Peng JH, Luo XQ, Yin RX, Nguyen CP, Pan SL. Enrichment of MTHFR $677 \mathrm{~T}$ in a Chinese long-lived cohort and its association with lipid modulation. Lipids Health Dis. 2014;13:104.

38. Jain M, Pandey $P$, Tiwary NK, Jain S. MTHFR C677T polymorphism is associated with hyperlipidemia in women with polycystic ovary syndrome. J Hum Reprod Sci. 2012;5:52-6.

39. Huang L, Song XM, Zhu WL, Li Y. Plasma homocysteine and gene polymorphisms associated with the risk of hyperlipidemia in northern Chinese subjects. Biomed Environ Sci. 2008;21:514-20.

40. Chauhan G, Kaur I, Tabassum R, Dwivedi OP, Ghosh S, Tandon N, et al. Common variants of homocysteine metabolism pathway genes and risk of type 2 diabetes and related traits in Indians. Exp Diabetes Res. 2012. doi:10.1155/2012/960318.

41. Lee EJ, Cho YJ, Yoon YJ. Methylenetetrahydrofolate reductase C677T gene mutation as risk factor for sudden sensorineural hearing loss: association 
with plasma homocysteine, folate and cholesterol concentrations. J Laryngol Otol. 2010;124:1268-73.

42. Li WX, Lv WW, Dai SX, Pan ML, Huang JF. Joint associations of folate, homocysteine and MTHFR, MTR and MTRR gene polymorphisms with dyslipidemia in a Chinese hypertensive population: a cross-sectional study. Lipids Health Dis. 2015;14:101.

43. Wen C, Lv JF, Wang L, Zhu WF, Wan FS, Wang XZ. Association of a methylene tetrahydrofolate reductase C677T polymorphism with several blood chemical levels in a Chinese population. Genet Test Mol Biomarkers. 2015;19:24-9.

44. Yang B, Fan S, Zhi X, Wang D, Li Y, Wang Y, Wang Y, Wei J, Zheng Q, Sun G. Associations of MTHFR C677T and MTRR A66G gene polymorphisms with metabolic syndrome: a case-control study in Northern China. Int J Mol Sci. 2014;15:21687-702.

45. Misiak B, Laczmanski L, Sloka NK, Szmida E, Piotrowski P, Loska O, Slezak R, Kiejna A, Frydecka D. Metabolic dysregulation in first-episode schizophrenia patients with respect to genetic variation in one-carbon metabolism. Psychiatry Res. 2016;238:60-7.

46. Scicchitano P, Cameli M, Maiello M, Modesti PA, Muiesan ML, Novo S, et al. Nutraceuticals and dyslipidaemia: beyond the common therapeutics. J Funct Foods. 2014;6:11-32.

47. Yin RX, Li YY, Wu JZ, Pan SL, Liu CW, Lin WX, Yang DZ. Interactions between the apolipoprotein a1/c3/a5 haplotypes and alcohol consumption on serum lipid levels. Alcohol Clin Exp Res. 2013;37:234-43.

48. Yao MH, Guo H, He J, Yan YZ, Ma RL, Ding YS, Zhang JY, Liu JM, Zhang M, Li SG, Xu SZ, Niu Q, Ma JL, Guo SX. Interactions of Six SNPs in ABCA1gene and Obesity in Low HDL-C Disease in Kazakh of China. Int J Environ Res Public Health. 2016;13:176.

49. Ruixing Y, Yiyang L, Meng L, Kela L, Xingjiang L, Lin Z, et al. Interactions of the apolipoprotein C-III 3238C > G polymorphism and alcohol consumption on serum triglyceride levels. Lipids Health Dis. 2010;9:86.

50. Ko YL, Hsu LA, Hsu KH, Ko YH, Lee YS. The interactive effects of hepatic lipase gene promoter polymorphisms with sex and obesity on high-densitylipoprotein cholesterol levels in Taiwanese-Chinese. Atherosclerosis. 2004:172:135-42.

\section{Submit your next manuscript to BioMed Central and we will help you at every step:}

- We accept pre-submission inquiries

- Our selector tool helps you to find the most relevant journal

- We provide round the clock customer support

- Convenient online submission

- Thorough peer review

- Inclusion in PubMed and all major indexing services

- Maximum visibility for your research

Submit your manuscript at www.biomedcentral.com/submit

) Biomed Central 